\title{
LOW CODE PROGRAMMING WITH ORACLE APEX OFFERS NEW OPPORTUNITIES IN HIGHER EDUCATION
}

\author{
Alenka Baggia ${ }^{1}$ \\ Robert Leskovar $^{2}$ (D) \\ Blaž Rodič $\check{c}^{3}$
}

DOI: https://doi.org/10.31410/ITEMA.S.P.2019.91

\begin{abstract}
Rapid development of software development tools presents lecturers in ICT study programs with a demanding challenge: selecting optimal tools to be used in classrooms. An interesting option is low code development environments, where minimal programming skills are needed to develop simple, yet attractive web applications with responsive design. We present several cases of introducing such a tool - Oracle Application Express (APEX) at Faculty of Organizational Sciences at the University of Maribor study programs. Diverse approaches were used to bring APEX closer to students, from incorporating social network paradigm, development of web application, to software quality assurance. Based on the positive experience of using the APEX development environment in the classroom, other faculties are considering introducing similar tools in their study programs.
\end{abstract}

Keywords: Higher Education, ICT study programs, Low Code Development Environment, Web Applications, User Interface, Oracle APEX

\section{INTRODUCTION}

$\mathrm{H}$ igher education institutions offering ICT study programs have to face demanding challenges to compete for students that want to learn application development and usage of various software (SW) development tools. Apart from the influence of the rapid evolution of SW development tools on the curricula, faculties have to compete with a large number of selfpaced online learning platforms. Faculties aim to train students to be flexible and prepared to think and act out of the box to be able to compete in the job market. IT companies that follow the dynamics of the technological segments often consider fresh graduates unprepared for work (Babič, Gašpar, \& Satala, 2018).

This paper aims to present several case studies of the introduction of a low code development environment in study programs. Cases were implemented in diverse courses at the University of Maribor, Faculty of Organizational Sciences (UM FOV) and are planned at the Faculty of Information Studies (FIS). Both involved study programs are not strictly focused on computer science and programming but have a wider focus on the management of information systems, where a relatively less technical approach to application development proves to be very useful and efficient. The aim was to introduce the application development environment gradually, considering the needs of a particular study level.

The tool of choice in our cases was Oracle Application Express (APEX), both for its availability within the Oracle Academy initiative and its reputation as a suitable tool for the teaching and

\footnotetext{
University of Maribor, Faculty of Organizational Sciences, Kidričeva cesta 55a, SI-4000 Kranj, Slovenia University of Maribor, Faculty of Organizational Sciences, Kidričeva cesta 55a, SI-4000 Kranj, Slovenia Faculty of Information Studies, Ljubljanska cesta 31a, SI-8000 Novo Mesto, Slovenia
} 
learning of enterprise databases (Monger, Baron, \& Lu, 2009), as well as web application development (Mason, 2013)share their knowledge with peers and can inspire students to study the subject after they graduate from the university. One of the goals of the Regis database practicum is to inspire students to continue to study the subject of database technologies after they graduate (e.g., life long learning.

\section{ORACLE APPLICATION EXPRESS}

Oracle APEX enables the design, development, and deployment of database-driven applications using only a web browser (Oracle, 2018b). It combines the qualities of a low code tool such as productivity, ease of use, and flexibility, with the qualities of an enterprise development tool such as security, integrity, scalability, availability and built for the web (Oracle, 2017). It allows end-users to interact with their data via tools like the interactive report, interactive grid, different types of charts, maps and more (Riaz, 2018). When needed, developers can also include SQL, PL/SQL, HTML, JavaScript and CSS to enhance the user interface.

APEX is the native web development tool that is installed with the Oracle Database (Geller \& Spendolini, 2017). All the data presented, inserted or updated in the web application developed with Oracle APEX is therefore securely saved in the database. Most of the basic functionalities of APEX are supported by wizards, which lead the developer through the process of web application development. Consistent, user-friendly report pages, data entry forms or tabular forms are therefore developed with minimum coding effort. From the other perspective, when the usage of applications developed with Oracle APEX is considered, one of the main advantages of is a high degree of client-side platform independence (Geller \& Spendolini, 2017).

\section{ORACLE APEX IN THE EDUCATIONAL PROCESS}

The Oracle Academy Initiative offers an online course "Oracle Application Express - Application Development Foundations", which introduces students to the techniques and tools required to develop database-driven web applications (Oracle, 2018a). Students can gain specific Oracle Academy certificates for the successful completion of the online course. Although certification can be used as a diploma supplement when applying for a job, only some of the students involved in case studies were interested in applying for the certification procedure. According to previous experiences of the instructors, the student's lack of interest in the certification is usually caused by the lack of knowledge on certain topics. Therefore, the authors decided to familiarize students with Oracle APEX gradually during their study.

In the academic year 2014/15 Oracle APEX was used for the first time at UM FOV, Organization and management of information systems (OMIS) study program. A physical server with Oracle APEX 4.2 was installed and made available to the master's degree students. For the last three years, students have also been advised to use free cloud workspace at the apex.oracle.com website for their assignment and self-study.

\section{CASE STUDIES IN GRADUATE STUDY}

Oracle APEX was introduced in several courses at UM FOV professional and academic study programs. Case studies of individual courses are presented in this chapter. 


\subsection{Computer science and informatics course}

The obligatory course Computer science and informatics is carried out in the first year of study at the three study programs at UM FOV (Organization and management of information systems, Organization and management of human resources and educational systems, and Enterprise engineering). To encourage the students to connect with their classmates and at the same time learn about the web application development, they are invited to log in to the Oracle APEX application and enter data about their peers and the level of acquaintance. The lecturer develops a simple web application based on the social network paradigm in-vivo, at the lectures. Following this presentation, students are invited to log in and mark their acquaintances. The collected connections are gathered in a database table. Students are invited to log in and mark their acquaintances. Further on, graphical representation of top »knowers« and top known students is added to the interface. Students upgrade their experience on their social network at lab exercises, where they learn how to design and present the formed social network with $\mathrm{R}$ software (Figure 1).

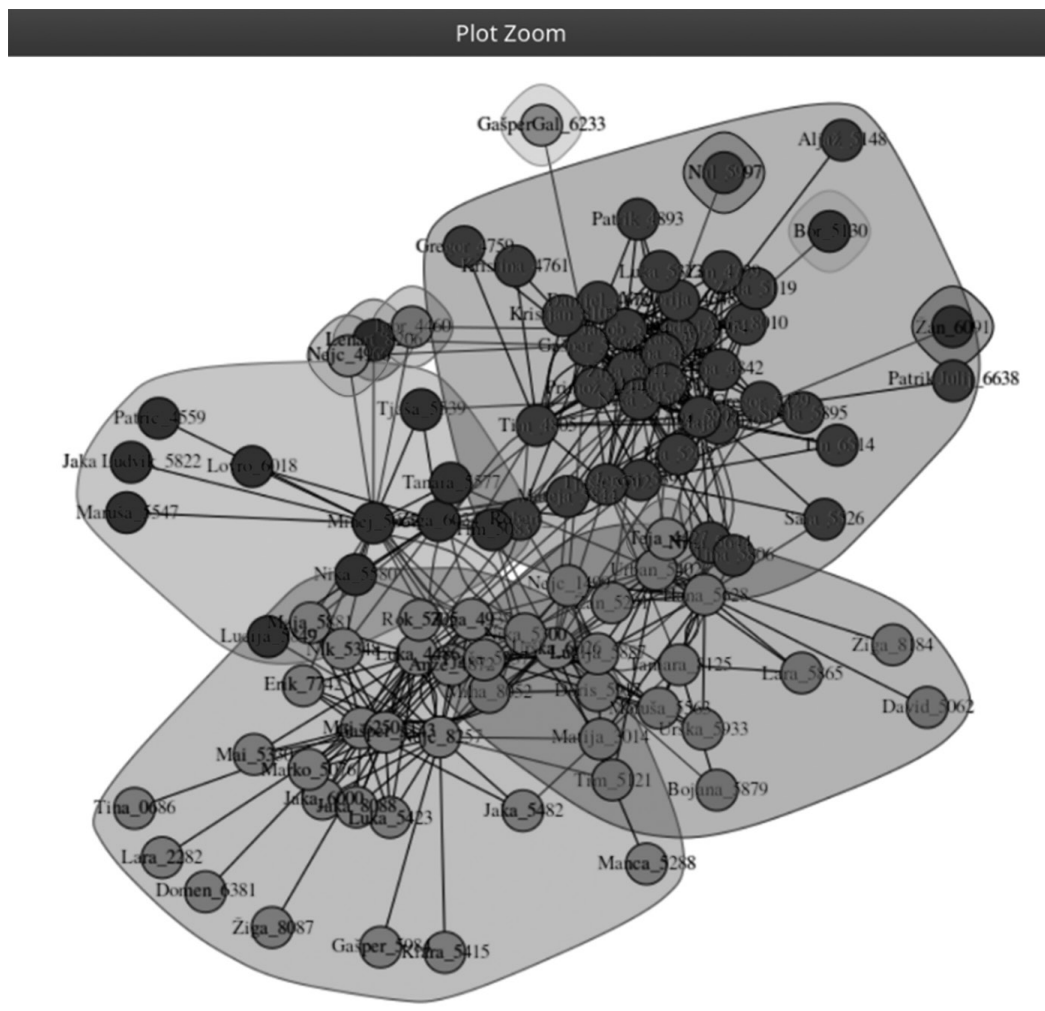

Figure 1. The social network of $1^{\text {st }}$-year students

\subsection{System analysis course}

In the second year of study, students attend the course System analysis that introduces them to the process of information systems development. In addition to process modelling techniques, students are introduced to database design and the basics of Structured Query Language (SQL). The goal of the course is to follow the information system (IS) design phases, producing a working prototype at the end of the course. Since Oracle database is used to practice SQL basics, students are encouraged to design a prototype in Oracle APEX low code environment. A simple example of employees and departments is used to present the basics for forms and interactive reports development (Baggia, Mali, Grlica, \& Leskovar, 2018). 
To enhance the knowledge and gain additional first-hand information, two expert APEX developers were invited to conduct a workshop in the study year 2017/18, The 3 - hour workshop was offered to the students in addition to regular lectures and lab practices. The developers used a simple case, which students proposed and developed partly based on their knowledge, to show some advanced techniques and new APEX capabilities. Due to the change of study program, in 2018/19 students were provided with web tutorials on basic APEX application development. Each student selected his/her own case of information system and developed a prototype application with a minimum of one master/detail form.

\subsection{IT project course}

In the last year of graduate study, students attend a specialized course, where they work in teams to develop an IT project based on the selected methodology. All the lecturers and assistants working at the OMIS study program are engaged as mentors in this course. The challenge for a certain team can be selected and defined by the students or selected by the mentor. Challenges should be real-world problems in an organization, where a prototype solution can be developed in the given time frame (6 months).

The number of team members depends on the number of students enrolled in the course, with a minimum of three members. In the study year, 2017/18 one team of nine students has applied their knowledge at the problem presented by an enterprise with 800 employees having issues with a lack of interest of employees for their intranet solution. Since the enterprise uses an Oracle database, a proposal was made to develop a prototype of an intranet application with Oracle APEX. After three meetings with the client (representatives of the enterprise), students formulated the specification for the updated intranet application and started with the prototype development in the Oracle APEX cloud.

The presentation of the prototype was held at the end of the study year. The representatives of the enterprise and all lecturers involved in the IS project course participated in the event. Several issues and suggestions for improvements were given to the students. One of the students decided to develop the prototype into a working application and use this topic as a graduate degree thesis. A page presenting the homepage of the intranet application and selection of documents and application forms as seen by a manager is shown in Figure 2.

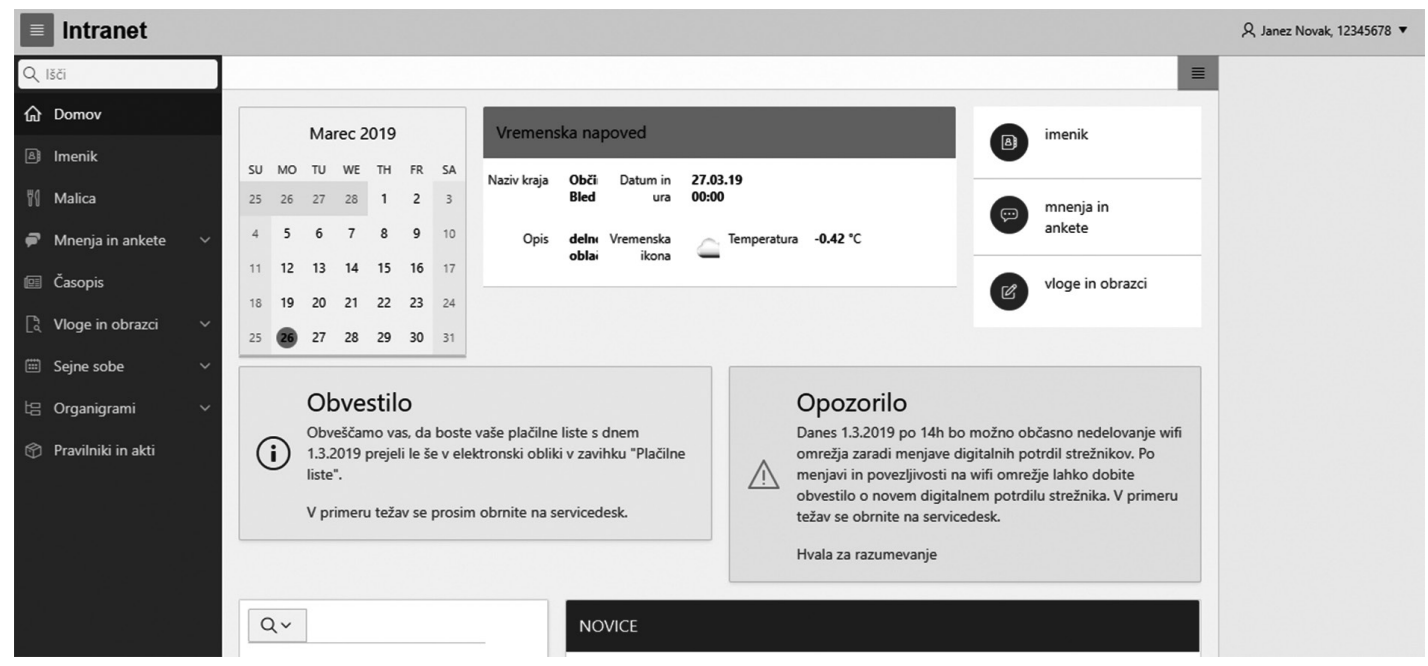

Figure 2. Intranet application (Mihelič, 2019) 


\section{CASE STUDIES IN MASTER DEGREE STUDY}

\subsection{Software quality course}

In the second year of master's degree study, students are enrolled in the course Software Quality, where they get an insight into software quality assurance in the process of IS development. In the study year 2017/18, students were given the challenge to develop an application with an emphasis on software quality assurance in each development phase. Students worked in teams, which were formed after a detailed analysis of prior knowledge. Due to the diversity of background knowledge of master's degree OMIS students, usage of low code environment, Oracle APEX was proposed. In addition to the basics of software quality assurance, students were introduced to the Oracle APEX development environment. Basics of APEX can be learned very quickly, whereas profound usage requires more training and knowledge.

Students were given the challenge to develop an application, which can be used to collect feedback at the lectures from students' mobile devices. When appropriate, the lecturer enters the question and students have to reply to the question in a predefined time (usually 30 to 180 seconds). Due to the mentioned diversity of knowledge, students were invited to play different roles in the development process. Students with limited knowledge in programming and application development were therefore asked to develop a detailed specification for the proposed application following the life cycle methodology. Coders were asked to write comments to their code, to enable easier testing procedures. To ensure the proper functioning of the database objects, unit testing was performed in SQL Developer. Furthermore, a simple web application was developed using Oracle APEX. The application enables the lecturer to interact with the student by posting challenges to which a student has to respond. Based on ISO/IEC/IEEE 29119 standard (ISO/IEC/ IEEE, 2014), a testing plan for the application was formulated, resulting in the testing report.

Students presented their prototype as a poster at the 2018 Apex Alpe Adria conference in Graz, where their work was introduced to the Senior Director for Software Development at Oracle, Mr. Joel R. Kallman (Figure 3).

Joel R. Kallman @joelkallman·Apr 20

Very honored to meet the faculty and students from University of Maribor (an

@OracleAcademy educational institution) who use @OracleDatabase, PL/SQL and

@OracleAPEX in multiple university classes. \#orclapex \#aaapeks18
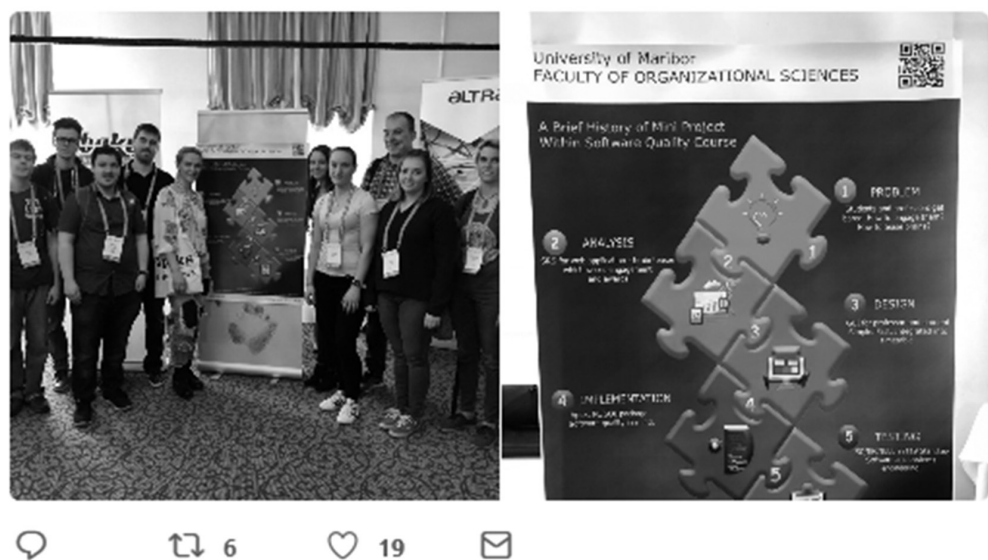

Figure 3. UM FOV master degree students meeting Mr. Joel R. Kallman, Senior Director,

Software Development at Oracle America, Inc. 
In the study year 2018/19 the students received a special challenge in the form of a project assignment, aiming to unify the existing IT solutions using APEX. In recent years several APEX based user interfaces and applications have been created within various study courses, with each application using a separate database. As the databases were closely related in content, we have decided to consolidate the databases and their respective user interfaces or applications. A total of six applications and their databases were included in the project assignment.

The project assignment was specified as such: the design of current databases allows multiple applications to be used but requires the user to switch between different Apex workspaces, leading to poor user experience. As the database structure and program code in packages are related, it is possible to consolidate data and applications. The assignment goals were:

1. Create a new, consolidated workspace in APEX,

2. Review the existing code in individual workstations,

3. Create and test a new script for creating tables, insights, sequences, triggers, including all foreign keys,

4. Create a new, consolidated database with all relevant tables, sequence views, and triggers,

5. Transfer relevant existing and new data into the new database,

6. Schedule information was provided in the spreadsheet. The data had to be rearranged, prepare the lookup fields that they were in the appropriate format for entering the database.

7. Create and test a new PKG_PREDAVALNICA package with consolidated and tested functions and procedures,

8. Transfer existing applications and update them with new features,

9. Test the software,

10. Prepare project documentation.

Students worked on the fixes and updates of both the database and the applications in groups, under the mentorship of the professor or assistant. They conducted a complete code review, entered the relevant comments in the code, and checked the appropriateness of the naming tables and other elements used in the code. They used the Trivago PL/SQL Cop tool to view the PL/ SQL code and obtain suggestions for improvements.

As a result, the students created one common database and updated three different applications. Students had to use a new, updated database, and import it into their APEX Workspace. Due to the complexity of ISO/IEC/IEEE 29119, the test plans were simplified. All tests and incident reports have been compiled into the documentation that will be used by the next generation of students to update the applications.

\section{CONCLUSION}

Oracle APEX is a low code environment that comes with Oracle database and can be used to develop enhanced web applications with some coding input, as well as simple web applications with minimal knowledge and skills in programming. The presented case of using Oracle APEX at the University of Maribor, Faculty of Organizational Sciences shows, that APEX can be used in different educational levels, from secondary school to master degree study in the field of information systems. The main advantage of APEX is that it can be used for topics not strictly related to IS development, as well as advanced topics in IS education. In addition, web application development with APEX gives students an opportunity to get real experience with working in the cloud. 
Since Oracle Academy Database design and foundations, SQL and PL/SQL courses are available to UM FOV students, they are also encouraged to extend their knowledge and earn certificates of course completion for Oracle APEX. Based on the good experience using Oracle free workspace service for the development of APEX applications in the study process, this approach will also be used in the following years. Whereas for advanced projects, like IT project course, on-premises installation of Oracle APEX will ensure better performance and more space to save the data. In addition to activities integrated into the courses and Oracle Academy certifications, students are also invited to participate in workshops organized by the Oracle APEX special interest groups (e.g. Apex Alpe Adria).

Faculty's experiences with low code application development environments have been presented on several occasions, with other HE institutions showing interest. Due to the positive experiences with Oracle APEX at UM FOV, Faculty of Information Studies in Novo Mesto is also looking to introduce Oracle APEX as a low code application development environment within their first level study programs in courses Web and Mobile Information Systems and Development of Solutions for Small Companies. Their use case will be documented and presented in further publications.

\section{REFERENCES}

Babič, F., Gašpar, V., \& Satala, P. (2018). New Trends in Mobile Technologies Education in Slovakia: An Empirical Study. ICETA 2018 - 16th IEEE International Conference on Emerging E-Learning Technologies and Applications, Proceedings, 37-42. https://doi. org/10.1109/ICETA.2018.8572277

Baggia, A., Mali, A., Grlica, A., \& Leskovar, R. (2018). Oracle APEX in Higher Education. In O. Arsenijević, I. Podbregar, P. Šprajc, D. Trivan, \& Y. Ziegler (Eds.), Proceedings of the 37th International Conference on Organizational Sciences Development (pp. 27-38). https://doi.org/10.18690/978-961-286-146-9

Geller, A., \& Spendolini, B. (2017). Oracle Application Express: Build Powerful Data-Centric Web Apps with APEX 5. New York: McGraw-Hill Education.

ISO/IEC/IEEE. (2014). ISO/IEC/IEEE 29119 Software Testing: the international standard for software testing. ISO/IEC/IEEE.

Mason, R. T. (2013). A Database Practicum for Teaching Database Administration and Software Development at Regis University. Journal of Information Technology Education: Innovations in Practice, 12, 159-168.

Monger, A., Baron, S., \& Lu, J. (2009). More on Oracle APEX for Teaching and Learning. Ideas in Teaching, Learning and Assessment of Databases: A Communication of the 7thInternational Workshop on Teaching, Learning and Assessment of Databases (TLAD 2009).

Oracle. (2017). What is Application Express?

Oracle. (2018a). Oracle Academy Courses.

Oracle. (2018b). Oracle Application Express.

Riaz, A. (2018). Oracle APEX 18.1 For Beginners: A Platform to Rapidly Develop Data-centric Web Applications Accessible from a Multitude of Devices. USA: CreateSpace Independent Publishing Platform. 